\title{
MRI Study of Nucleation and Growth During the Field Induced First Order Phase Transition Between U2D2 and CNAF 3He near Absolute Zero
}

\author{
$\operatorname{AUTHOR}(S):$ \\ Tanaka, T.; Yatsuya, M.; Mizusaki, T.; Sasaki, Y.
}

\section{CITATION:}

Tanaka, T....[et al]. MRI Study of Nucleation and Growth During the Field Induced First Order Phase Transition Between U2D2 and CNAF 3He near Absolute Zero. Journal of Low Temperature Physics 2010, 158(5-6): 737-748

ISSUE DATE:

2010-03

URL:

http://hdl.handle.net/2433/120331

\section{RIGHT:}

The original publication is available at www.springerlink.com; この論文 は出版社版でありません。引用の際には出版社版をご確認ご利用くだ さい。; This is not the published version. Please cite only the published version. 


\title{
MRI Study of Nucleation and Growth during the Field Induced First Order Phase Transition between U2D2 and CNAF ${ }^{3} \mathrm{He}$ near Absolute Zero
}

\author{
T. Tanaka ${ }^{a}$, M. Yatsuya $^{a}$, T. Mizusaki ${ }^{a, b}$, and Y. Sasaki ${ }^{a, c}$ \\ ${ }^{a}$ Department of Physics, Graduate School of Science, \\ Kyoto University, Kyoto 606-8502, Japan \\ ${ }^{b}$ Toyota Riken, Nagakute-cho, Aichi 480-1192, Japan \\ ${ }^{c}$ Research Center for Low Temperature and Materials Sciences, \\ Kyoto University, Kyoto 606-8502, Japan
}

(Dated: October 3, 2009)

\begin{abstract}
The field-sweep-induced first order phase transition between two nuclear antiferromagnetic states of solid ${ }^{3} \mathrm{He}$ was studied at the temperatures near absolute zero. The phase transition proceeded in two stages. The first stage was governed by a nucleation process, in which many small seeds of the stable phase appeared in many places throughout a crystal. The measured nucleation rate was essentially temperature independent, once we corrected the data for weak temperature dependence due to limited growth of the seeds after nucleation. With the help of MRI measurements, we understand that the nucleation occurs at peculiar heterogeneous nucleation sites, which are distributed in the entire crystal. Magnetic planar defects in the nuclear ordered spin structure are proposed as a candidate for the nucleation sites. The second stage is understood as a process, which is controlled by the flow of released latent heat across the solid-liquid interface.
\end{abstract}

PACS numbers: 64.60.Q-, 67.80.D-, 67.80.dk, 75.45.+j, 76.60.Pc 


\section{INTRODUCTION}

The first order phase transition at finite temperature is mostly understood to be initiated by the nucleation of a stable phase driven by thermal fluctuations. However the nucleation mechanism near absolute zero is not well understood. One possible explanation is a quantum nucleation process assisted by macroscopic quantum tunneling (MQT). Extensive studies have been made to show the quantum nucleation for various macroscopic systems, such as quantized vortices [1-3], cavitations [4], quantum crystals [5, 6]. They showed the saturation of the nucleation rate in a low temperature region, an effect understood to be a signature of crossover from thermal to quantum nucleation. To further understand the nature of quantum nucleation, it is important to study the nucleation rate as a function of an applied chemical potential difference. Some of them $[1,5,6]$ suggested that the nucleation occurred in a heterogeneous manner. However none of them made clear what was the heterogeneity. Studying the first order phase transition of solid ${ }^{3} \mathrm{He}$, a quantum crystal that has essentially no impurity in a crystal, has a significant advantage in studying nucleation centers. Motivated by these ideas, we studied the field induced first order phase transition between two nuclear ordered antiferromagnetic phases of solid ${ }^{3} \mathrm{He}$ at the temperature, $T$, well below $1 \mathrm{mK}$, at the critical magnetic field $B_{\mathrm{C} 1}[7]$. The low field phase has an up-updown-down (U2D2) spin structure, which has a uniaxial anisotropy along (100) axis of the bcc crystal. There is a huge NMR frequency shift due to the nuclear dipolar interaction. The high field phase has a canted normal antiferromagnetic (CNAF) spin structure, which has a cubic symmetry and much larger magnetization than the U2D2 phase. These features enable us to distinguish the two phases by the NMR measurement, and thus enable us to study the spatial distribution of the two phases in a crystal [8-10].

\section{EXPERIMENTAL PROCEDURE}

A seed crystal of U2D $2{ }^{3} \mathrm{He}$ was produced at the bottom of the $4 \mathrm{~mm}$ diameter cylindrical polycarbonate cell, which contained superfluid ${ }^{3} \mathrm{He}-\mathrm{B}$ at $0.5 \mathrm{mK}$ and was attached below the metallic compressional cell and heat exchangers, shown in Fig. 1. After growing a seed crystal into a single crystal of desired size, MRI measurement [13] was performed to check the distribution of magnetic domains in the crystal. Typically only three large magnetic 
domains appear at the bottom of the sample cell with flat domain boundaries between them. Temperature of the sample was obtained from the known temperature dependence of $B_{\mathrm{C} 1}$ [11], which was determined by the successive measurement of NMR spectrum while sweeping applied magnetic field $B$ slowly through $B_{\mathrm{C} 1}$. Since we observed no hysteresis on the measured $B_{\mathrm{C} 1}$ during magnetizing and demagnetizing measurement, the measured $B_{\mathrm{C} 1}$ was close enough to its thermoequilibrium value. CNAF phase has about 6 times larger magnetization than U2D2 phase and its free induction decay (FID) spectrum is broader in the vicinity of Larmor frequency because of the internal magnetic field, while spectrum of U2D2 phase divides into three sharp frequency-shifted peaks according to magnetic domains. Thus U2D2 phase and CNAF phase are easily distinguished by FID measurements. After achieving thermal equilibrium at $B$, which is just below $B_{\mathrm{C} 1}, B$ was quickly swept to $B=$ $B_{\mathrm{C} 1}+\Delta B$. A time evolution of the volume fraction of the U2D2 phase was obtained from intensities of the FID signals after small tipping angle $\left(\sim 1^{\circ}\right)$ pulses. Thanks to a rapid spin relaxation in the nuclear ordered phases, we could measure the FID signals with intervals as short as 0.2 second without affecting the spectrum. The volume fraction of the metastable U2D2 phase can be obtained as

$$
\delta m \equiv\left|M_{\mathrm{C}}-M_{o b s}(t)\right| /\left|M_{\mathrm{C}}-M_{\mathrm{U}}\right|
$$

where $M_{\mathrm{C}}$ is the signal intensity when the entire crystal is in the stable CNAF phase and $M_{\text {obs }}(t)$ is the observed signal intensity at time $t$ after the quick magnetic field sweep to $B=B_{\mathrm{C} 1}+\Delta B$. After the transition was completed in the CNAF phase, the reversed process to U2D2 phase was measured in the same manner with $\Delta B<0$. The volume fraction of the metastable CNAF phase can be derived by replacing $M_{\mathrm{C}}$ with $M_{\mathrm{U}}$, which is the signal intensity when the entire crystal is in the stable U2D2 phase, in the numerator of Eq. (1).

\section{TIME EVOLUTION OF A VOLUME FRACTION OF THE STABLE PHASE DURING PHASE TRANSITION}

As shown in Fig. 2, the phase transition proceeded in two stages. During the first stage, $\delta m$ decreased exponentially in time, as $\exp \left(-\gamma_{1} t\right)$. Then at $\delta m=\delta m_{t r}$, the first stage terminated and the slower second stage took over. Figure 3 shows $\Delta B$ dependence of the 
rate $\gamma_{1}$ at various temperatures. The $\gamma_{1}$ increased with increasing $|\Delta B|$, and decreased slightly with increasing temperature. Figure 4 shows $\Delta B$ dependence of $\delta m_{t r}$. The $\delta m_{t r}$ decreased with increasing $|\Delta B|$, and increased with increasing temperature. Neither of the quantities, $\gamma_{1}$ and $\delta m_{t r}$ depended on a size of a crystal.

Time evolution of the volume fraction of the stable phase during the first order phase transition behaves quite differently depending on whether the nucleation process dominates or the growth process dominates. In the case where growth process dominates, the time evolution behaves as $(v t)^{n}$, where $v$ is a velocity of moving interface between two phases, and the integer $n=1$ to 3 is the dimension of the growing seed. To explain the exponential time evolution in the growth dominated situation, one would need exponential time dependence on $v$. Since thermal conductivity in the U2D2 phase varies exponentially with temperature [14], the velocity $v$ may vary in time due to a temperature variation caused by the latent heat of the phase transition. However, the actual temperature variation is so small that the velocity cannot have exponential time dependence. Thus the exponential dependence on time suggests that the nucleation process controls the time evolution during the first stage. The nucleated seeds may grow; however, this growth should not control the time evolution of the volume fraction of the stable phase, since it will not give exponential time evolution. We understand the exponential time evolution of $\delta m$ during the first stage and the termination of the first stage at $\delta m_{t r}$ as follows. While a seed of stable phase grows in the surrounding metastable phase, the latent heat of the first order phase transition must be released. Then, a temperature of the region in which this growing seed of stable phase and surrounding shell of metastable phase are contained changes gradually towards the coexisting temperature $T_{\mathrm{C} 1}$ at $B=B_{\mathrm{C} 1}\left(T_{\mathrm{i}}\right)+\Delta B$, where $T_{\mathrm{i}}$ is the temperature before applying $\Delta B$, regardless of the direction of the phase transition. When the temperature of the moving interface between two phases reaches the coexisting temperature $T_{\mathrm{C} 1}(B)$, chemical potential difference, which is the driving force of the growing stable phase, disappears and the seed of stable phase stops growing. As a result, formation of a matured seed, which contains a seed of stable phase and a surrounding shell of metastable phase at the coexisting temperature $T_{\mathrm{C} 1}(B)$, is completed. We call this growth process as limited growth. Then another nucleated seed appears at a separate place and grows up to another matured seed in the same manner. This process continues until many matured seeds occupy the entire crystal. This is the end of the first stage. If a time scale of each limited growth of a seed is much shorter than the time 
scale of the first stage, nucleation process, which keeps occurring in many places throughout the first stage, dominates the time evolution and exhibits the exponential time dependence. A proposed temperature shift towards the coexisting temperature $T_{\mathrm{C} 1}(B)$ during the first stage was confirmed by the measurement of the NMR spectrum of U2D2 phase, which has known monotonic temperature dependence. A volume fraction $\alpha$ of the stable phase in the matured seed can be estimated as follows. For simplicity, we assume that the temperature inside of the region that we call the 'matured seed' is uniform and is thermally isolated from the surrounding. The entropy conservation within the region gives

$$
S_{\mathrm{m}}\left(T_{\mathrm{i}}\right)=\alpha S_{\mathrm{s}}\left(T_{\mathrm{f}}\right)+(1-\alpha) S_{\mathrm{m}}\left(T_{\mathrm{f}}\right),
$$

where $S_{\mathrm{m}}=a_{\mathrm{m}} T^{3}$ and $S_{\mathrm{s}}=a_{\mathrm{s}} T^{3}$ represent the entropy of metastable phase and stable phase, which are U2D2 phase and CNAF phase for the case of $\Delta B>0$, with corresponding coefficients $a_{\mathrm{m}}$ and $a_{\mathrm{s}}, T_{\mathrm{i}}$ and $T_{\mathrm{f}}$ are the temperatures before and after applying $\Delta B$. The $\alpha$ can be expressed as

$$
\alpha=\frac{\left(T_{\mathrm{i}} / T_{\mathrm{f}}\right)^{3}-1}{a_{\mathrm{s}} / a_{\mathrm{m}}-1} .
$$

The stable phase stops growing when $T_{\mathrm{f}}$ reaches the temperature on the coexistence line between two phases, where $B_{\mathrm{C} 1}\left(T_{\mathrm{f}}\right)=B_{\mathrm{C} 1}\left(T_{\mathrm{i}}\right)+\Delta B$. With the aid of numeric representation of the coexistence line, $B_{\mathrm{C} 1}(T)=0.452-0.092 T^{4}\left[\mathrm{mK}^{4}\right]$ given by Xia et al. [11], the $\alpha$ can be obtained as a function of $T_{\mathrm{i}}$ and $\Delta B$ :

$$
\alpha=\left\{\begin{array}{ll}
1.1 & (\Delta B>0) \\
-2.1 & (\Delta B<0)
\end{array}\right\} \times\left(\left(1-\frac{\Delta B[\mathrm{mT}]}{92 T_{i}^{4}\left[\mathrm{mK}^{4}\right]}\right)^{-\frac{3}{4}}-1\right)
$$

where numeric values, 1.1 and -2.1 , in the first term are obtained from the values of spin wave velocities, $5.0 \mathrm{~cm} / \mathrm{s}$ in the U2D2 phase and $7.8 \mathrm{~cm} / \mathrm{s}$ in the CNAF phase given in Ni et al. [12] and $a_{\mathrm{s}(\mathrm{m})} \propto n_{\mathrm{s}(\mathrm{m})} / v_{\mathrm{s}(\mathrm{m})}^{3}$, where $n_{\mathrm{s}(\mathrm{m})}$ is the number of spin wave modes in the stable (metastable) phase and $v_{\mathrm{s}(\mathrm{m})}$ is the spin wave velocity in the stable (metastable) phase. Since $\delta m_{t r}$ is a volume fraction of the metastable phase at the end of the first stage, $\delta m_{t r}$ is $1-\alpha$. The estimated $1-\alpha$ is drawn in Fig. 4, which gives reasonable agreement with the experimental value of $\delta m_{t r}$ for each temperature. 


\section{TEMPERATURE AND $\triangle B$ DEPENDENCES OF $\gamma_{1}$}

Next, we consider the negative temperature dependence of the rate $\gamma_{1}$. The first order phase transition has two kinds of nucleation mechanism. One is to overcome the potential barrier with the aid of either thermal activation or MQT. The other is a spinodal region nucleation, which is caused by a disappearance of barrier due to an increased chemical potential difference between two phases. In the former case with thermal activation, the nucleation rate should be proportional to $\exp \left(-\Delta U / k_{B} T\right)$, where $\Delta U$ denotes the height of the potential barrier. It is quite unlikely that $\Delta U$ depends on temperature, when the physical origin of $\Delta U$ is the exchange energy. Thus the temperature dependence of a nucleation rate in the case of thermal activation is more or less $\exp (-1 / T)$. This strong, positive temperature dependence will not agree with that of the measured $\gamma_{1}$. In the case of MQT, a nucleation rate is temperature independent basically. It is possible to have temperature dependence in a special case when $\Delta U$ is temperature dependent or an effect of dissipation during tunneling is temperature dependent. However this is quite unlikely.

We explain the measured temperature dependence of $\gamma_{1}$ as follows. The measured $\gamma_{1}$ is a rate constant of a volume of metastable phase, while the real nucleation rate $\gamma_{n u c}$ is a rate constant for the number of nucleation sites. According to our scenario of the limited growth after nucleation, a nucleated seed grows and stops growing when it becomes a matured seed. Let the linear dimension of the matured seed be $\eta$ and the mean distance between nucleation sites be $\zeta$. If $\eta<\zeta, \gamma_{1}$ coincides with $\gamma_{n u c}$. In this case we have a difficulty to understand the temperature dependence as we discussed above. In the case of $\eta>\zeta$, one nucleated seed grows and absorbs many other nucleation sites located nearby until it becomes a matured seed. Then the measured rate $\gamma_{1}$ will be $n_{0} \gamma_{n u c}$, where $n_{0}$ is the number of absorbed nucleation sites. In this case, temperature dependence of $n_{0}$ contributes to the temperature dependence of $\gamma_{1}$. Assuming the limited growth is isotropic in 3 dimension, $n_{0}$ is equal to $(\eta / \zeta)^{3}$. Temperature dependence of $n_{0}$ comes from that of $\eta$, which can be estimated as follows. The spin system in the U2D2 phase has four sublattices, while that of CNAF phase has two sublattices. This means that for each magnetic-unit-cell-sized step of the interface between two phases spin flipping must occur. Due to this spin flipping associated with the interface motion, a magnon, of which wave number is as high as the zone-boundary value and hence its energy is as high as that of the zone-boundary magnon 
in the first Brilloin zone, must be emitted at the moving interface. Thermalization occurs through the Umklapp collision of this high-energy magnon, which is schematically shown in Fig. 5. When the mean free time of the magnons between Umklapp collisions is $\tau_{\mathrm{U}}, \eta$ can be estimated as $v_{\text {int }} \tau_{\mathrm{U}}$, where $v_{\text {int }}$ is a velocity of the moving interface. This velocity $v_{\text {int }}$ can be as high as the magnon velocity in the collisionless regime. We approximate $v_{\text {int }}$ by the spin wave velocity $c_{s}$, which is known to be temperature independent. No other length scale such as the thermal diffusion length should be used as a measure for $\eta$, since all the process is in the collisionless regime. Thus the temperature dependence of $\eta$ is given by the temperature dependence of $\tau_{\mathrm{U}}$. The scattering rate of the Umklapp process, where the high energy magnon with momentum q splits into two magnons, one of which has a small momentum $-\mathbf{q}^{\prime}$ and the other has a large momentum $\mathbf{q}+\mathbf{q}^{\prime}$ outside of the first Brilloin zone, can be expressed as

$$
\tau_{U}^{-1} \propto \iint\left(1+n_{-\mathbf{q}^{\prime}}\right)\left(1+n_{\mathbf{q}+\mathbf{q}^{\prime}}\right) d \varphi^{\prime} \mathbf{d} \mathbf{q}
$$

where $n_{\mathbf{q}}=\frac{1}{\exp \epsilon_{\mathbf{q}} / k_{\mathbf{B}} T-1}$ is the density of states of a magnon with momentum $\mathbf{q}$ and the corresponding energy $\epsilon_{\mathbf{q}}, \varphi^{\prime}$ is the energy-conservation surface (the locus of allowed values of $\left.\mathbf{q}^{\prime}\right)$. The quantity $\left(1+n_{-\mathbf{q}^{\prime}}\right)$ gives linear temperature dependence because $\epsilon_{-\mathbf{q}^{\prime}} \lesssim k_{\mathbf{B}} T$, while $\left(1+n_{\mathbf{q}+\mathbf{q}^{\prime}}\right) \simeq 1$. Thus $\tau_{U}$ is inversely proportional to temperature $T$. As a result $n_{0}$ is proportional to $T^{-3}$ and then the temperature dependence of $\gamma_{n u c}$ is given as $\gamma_{1} T^{3}$. Figure 6 shows the quantity $\gamma_{1} T^{3}$ as a function of $\Delta B$ for various temperatures. As can be seen from the figure, $\gamma_{n u c}$ is temperature independent. The scatter in the region $\Delta B>7 \mathrm{mT}$ is due to $\alpha$ approaching unity and hence not a problem. From these considerations, we conclude that the observed temperature dependence of $\gamma_{1}$ is given by the temperature dependence of $n_{0}$, and $\gamma_{n u c}$ is temperature independent.

Next, we consider the $\Delta B$ dependence of $\gamma_{1}$. A standard homogeneous nucleation model with an energy barrier, which is given by the interfacial energy and the chemical potential difference between two phases, predicts very strong chemical potential dependence of the nucleation rate, such as $\exp \left(-1 / \Delta B^{n}\right)$, where $n$ is a number of order unity. Lines in Fig. 6 show the strong chemical potential dependence of this model. As can be seen, this model is not adequate to explain the measured nucleation rate. Even for the heterogeneous nucleation near a lability boundary [5], the nucleation rate should have exponential dependence on $\left(\Delta B_{c}-\Delta B\right)$, where $\Delta B_{c}$ gives the lability boundary. This is still too strong dependence 
compared to what we observed. Thus we understand that the nucleation over an energy barrier is not the case. As a result we must think that, this is most likely the case of spinodal region nucleation associated to some heterogeneity, or in the other word instability, whose lability boundary $\left|\Delta B_{c}\right|$ is less than $0.5 \mathrm{mT}$. Even in such a case we require that the nucleation rate to be $\gamma_{n u c} \propto|\Delta B|$. Figure 7 shows the MRI image [13] of U2D2 phase soon after the first stage is terminated. This result shows that the nucleated seeds appeared everywhere in the crystal. Thus heterogeneous nucleation centers exist throughout the crystal. More surprisingly, the nucleation rate has similar value for both directions of the phase transition. This is contradictory to the idea of heterogeneous nucleation, since a heterogeneity, which favors one phase, is most probably unfavorable to the other phase.

\section{DISCUSSION}

At the ultra low temperatures of this experiment, solid ${ }^{3} \mathrm{He}$ has no impurities, thus the heterogeneity cannot be associated with impurities. We propose a possible candidate for this strange nucleation center. There are huge amount of linear crystalline defects, which are associated with planar magnetic defects in the U2D2 and CNAF antiferromagnet. As can be seen in Fig. 8, in the U2D2 phase, a missing plane [14] of up spins associated with edge dislocation gives a local down-up-down spin sequence, when the plane is perpendicular to the $\hat{\ell}$ axis, which is an anisotropy axis in the U2D2 phase, and thus may become a natural source of the CNAF spin structure. In the CNAF phase, a similar missing plane gives an up-downdown-up spin sequence and may become a natural source of the U2D2 spin structure with $\hat{\ell}$ axis perpendicular to the plane. Due to those magnetic planar defects, two antiferromagnets with very different symmetry may transform to each other through a very low nucleation barrier, which disappeared even with smallest $\Delta B \simeq 0.5 \mathrm{mT}$. Thus we observed weak and equivalent $\Delta B$ dependence for both direction of the phase transition. This may also explain the mystery of "Memory effect" [15], where the U2D2 domain distribution recovers after traveling back and forth between two phases.

The behavior of the second stage was rather complicated. It depended on the size of crystal. Figure 9 shows the MRI image during the second stage. It shows spatially inhomogeneous recovery from liquid-solid interface side, as is reported previously [8]. Thus we believe that the second stage can be understood as the thermal relaxation process, where 
the temperature change caused by the rapid latent heat release during the first stage relaxes through the heat flow across liquid-solid interface.

We conclude that the first stage of the field-sweep induced phase transition between U2D2 phase and CNAF phase proceeds through the heterogeneous nucleation through instability with limited-growth after the nucleation. The heterogeneous nucleation sites are possibly related to the magnetic planar defects associated with the crystalline linear defects.

This work was supported by Grant-in-Aid for Scientific Research on Priority Area "Physics of new quantum phases in superclean materials" (Grant No. 17071004), Grantin-Aid for the 21st Century COE "Center for Diversity and Universality in Physics" and Grant-in-Aid for the Global COE "The Next Generation of Physics, Spun from Universality and Emergence" from the Ministry of Education, Culture, Sports, Science and Technology of Japan.

[1] E. Varoquaux, W. Zimmerman, and O. Avenel, Excitations in 2D and 3D quantum fluids, A. F. G. Wyatt and H. J. Lauter (eds.), Plenum Press, New York (1991), p. 343 and references therein.

[2] J. C. Davis et al., Phys. Rev. Lett. 69, 323 (1992).

[3] G. G. Ihas et al., Phys. Rev. Lett. 69, 327 (1992).

[4] S. Balibar et al., J. Low Temp. Phys. 113 , 459 (1998) and references therein.

[5] J.P. Ruutu et al., Phys. Rev. Lett. 77, 2514 (1996).

[6] Y. Sasaki and T. Mizusaki, J. Low Temp. Phys. 110, 491 (1998).

[7] M. Roger, J.H. Hetherington, and J.M. Delrieu, Rev. Mod. Phys. 55, 1 (1983).

[8] T. Tanaka, H. Ito, Y. Sasaki, and T. Mizusaki, J. Phys. Chem. Solids 66, 1475 (2005).

[9] T. Tanaka, H. Ito, Y. Sasaki, and T. Mizusaki, AIP Conference Proceedings 850, 305 (2006).

[10] T. Tanaka, M. Yatsuya, Y. Sasaki, and T. Mizusaki, J. Mag. Mag. Mat. 310, 1615 (2007).

[11] J. S. Xia, W. Ni, and E. D. Adams, Phys. Rev. Lett. 70, 1481 (1993).

[12] W. Ni , J. S. Xia, and E. D. Adams, Phys. Rev. B 50, 336 (1994).

[13] Y. Sasaki, E. Hayata, T. Tanaka, H. Ito, and T. Mizusaki, J. Low Temp. Phys. 138, 911 (2005).

[14] Y. P. Feng, P. Schiffer, and D. D. Osheroff, Phys. Rev. B 49, 8790 (1994). 
[15] T. Ueno, Y. Kawaguchi, Y. Kinoshita, Y. Sasaki, and T. Mizusaki, J. Low Temp. Phys. 127, 1 (2002).

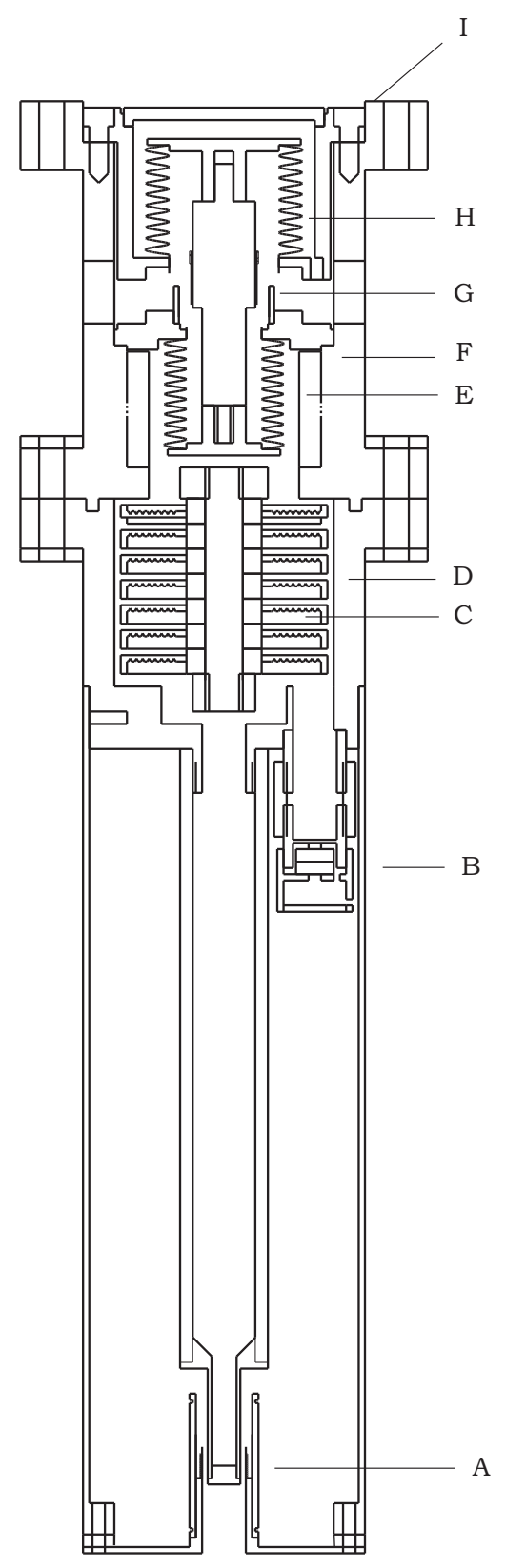

FIG. 1: Sample cell schematics. A: NMR receiver and transmitter coils, B: Capacitive Pressure Sensor, C: Sintered silver heat exchangers, D: Silver body, E: Sintered silver heat exchanger, F: Silver body, G: Capacitive displacement sensor, H: ${ }^{4} \mathrm{He}$ chamber, I: Thermal contact to nuclear stage 


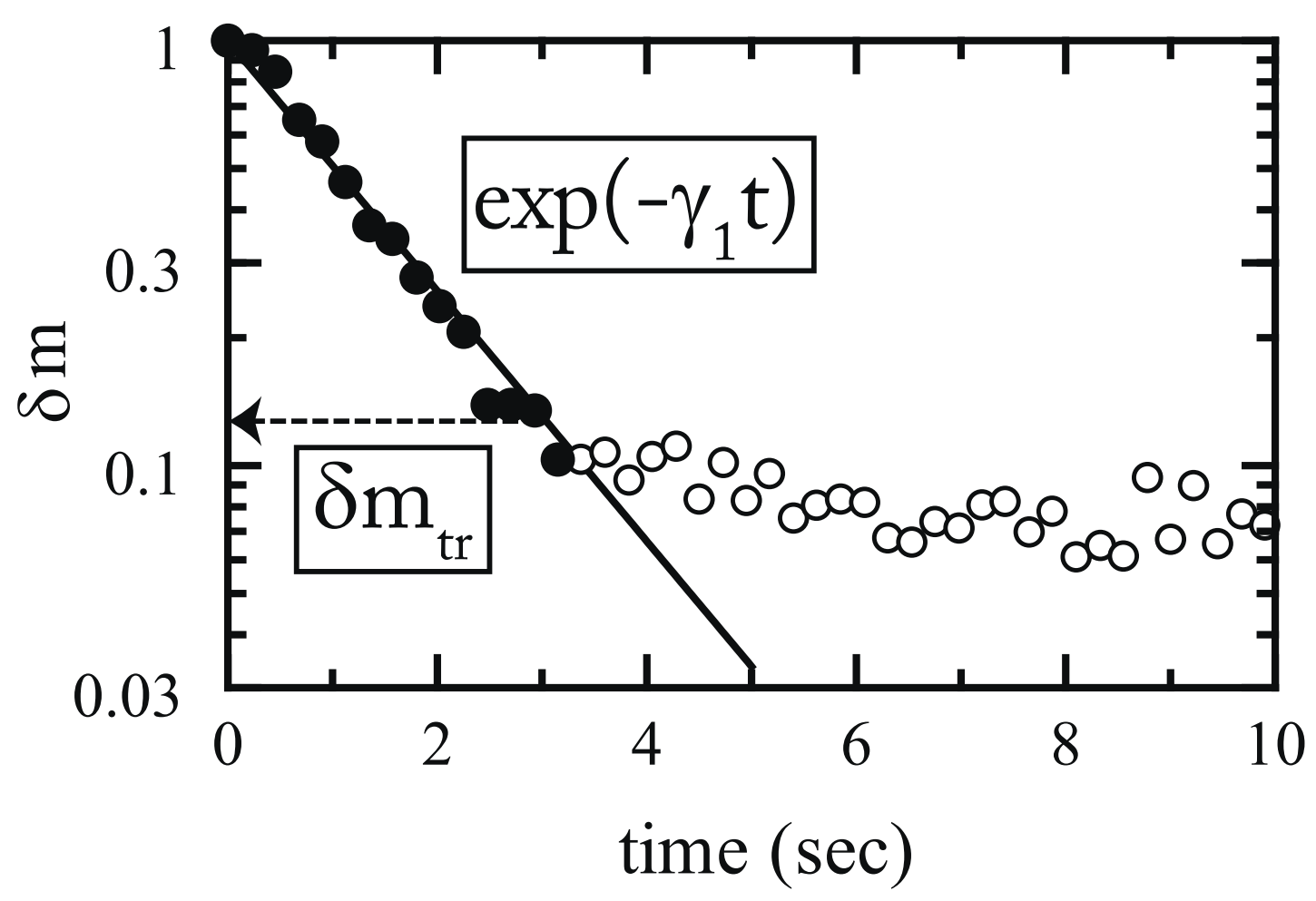

FIG. 2: Typical two stage time evolution of a volume fraction of the metastable phase $\delta m$ at $T=0.60 \mathrm{mK}$ and $\Delta B=-10 \mathrm{mT}$. 


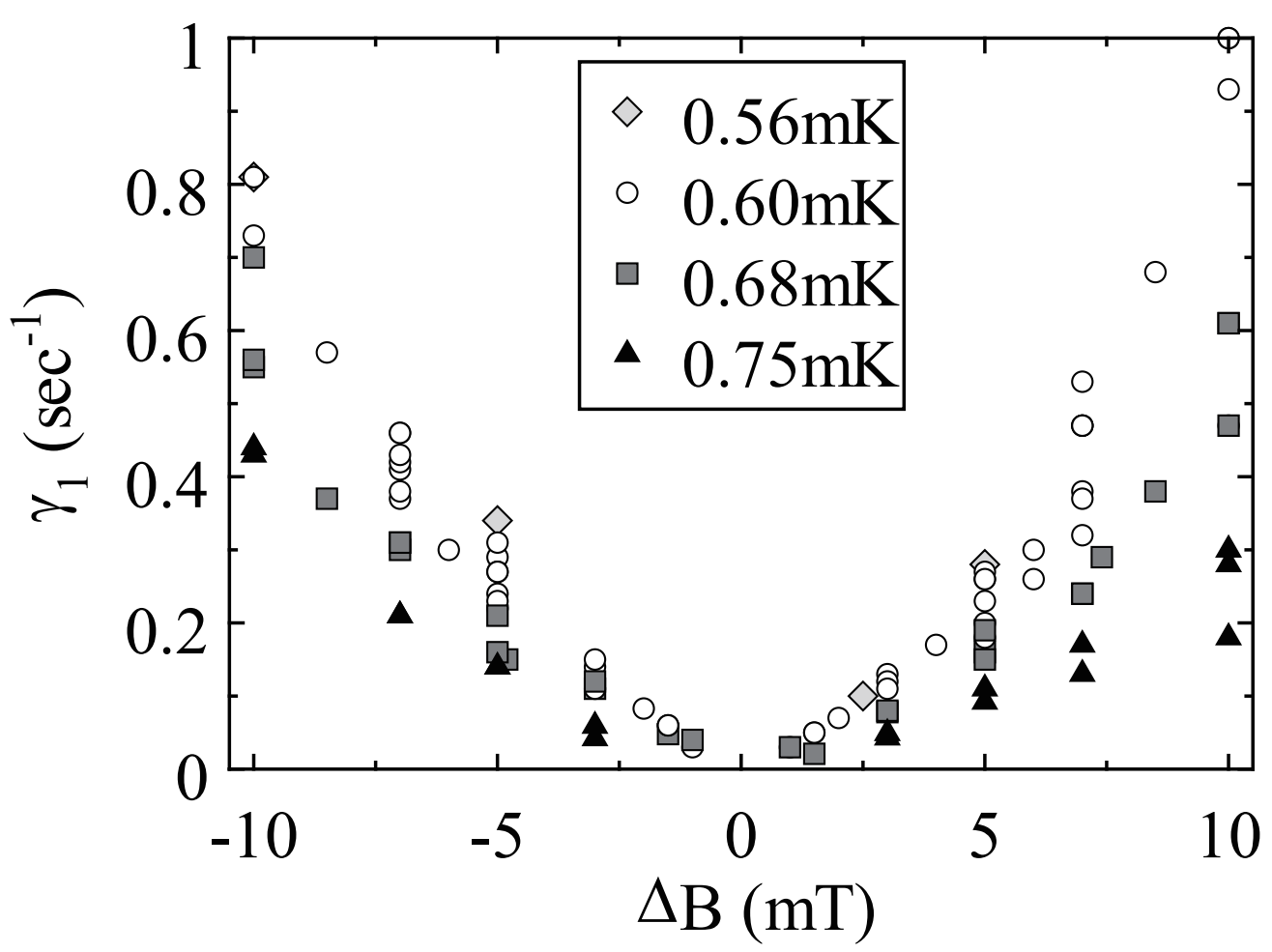

FIG. 3: The $\Delta B$ dependence of the measured rate $\gamma_{1}$ at various temperatures. 


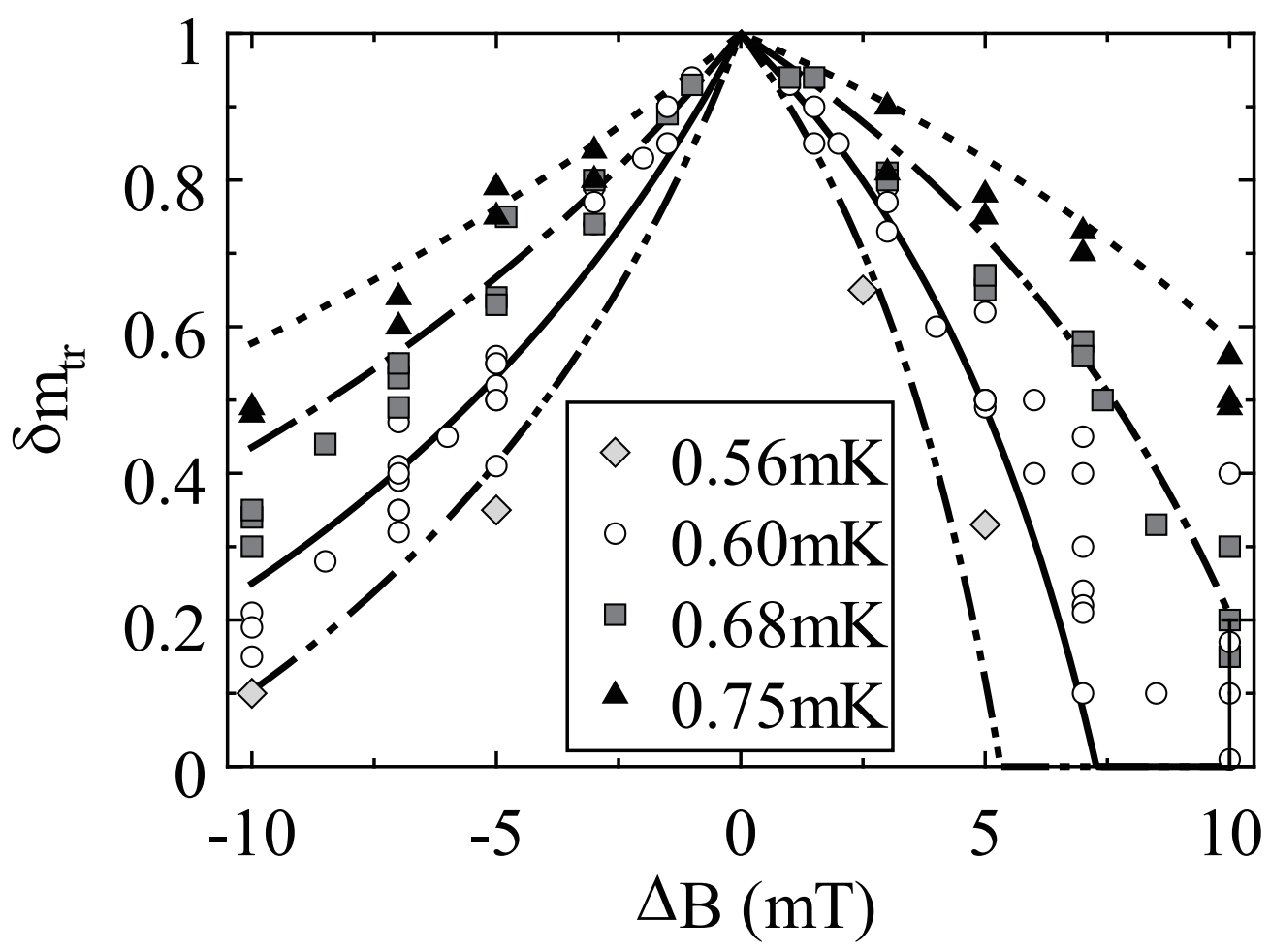

FIG. 4: The $\Delta B$ dependence of the $\delta m_{t r}$ at various temperatures. Lines are calculated by the model described in the text. 
(a)

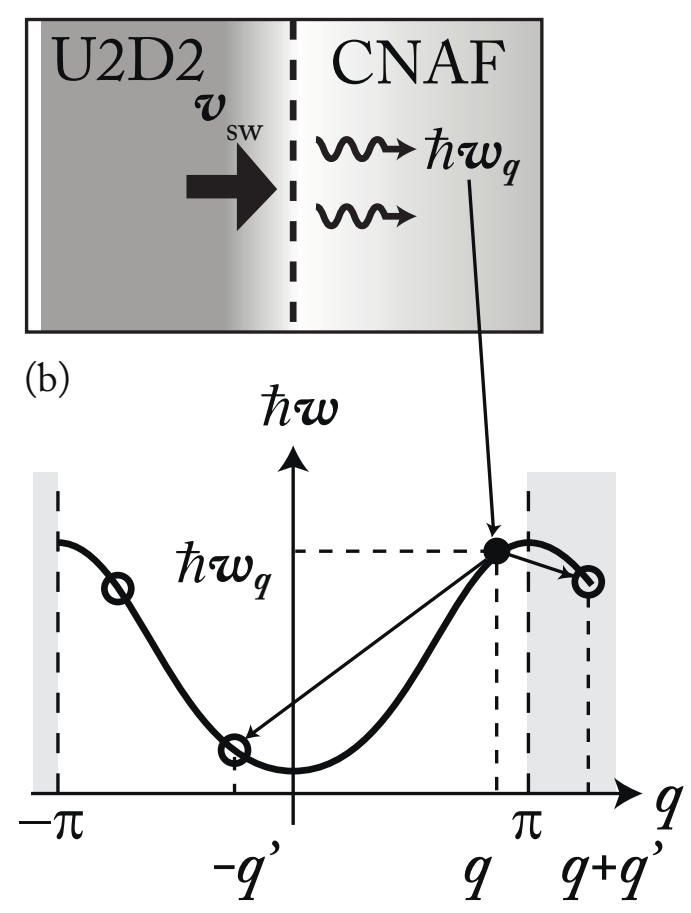

FIG. 5: (a): Schematic figure of the interface moving at the spin wave velocity and high-energy magnons emitted. (b): Schematic figure of three-magnon Umklapp process. 


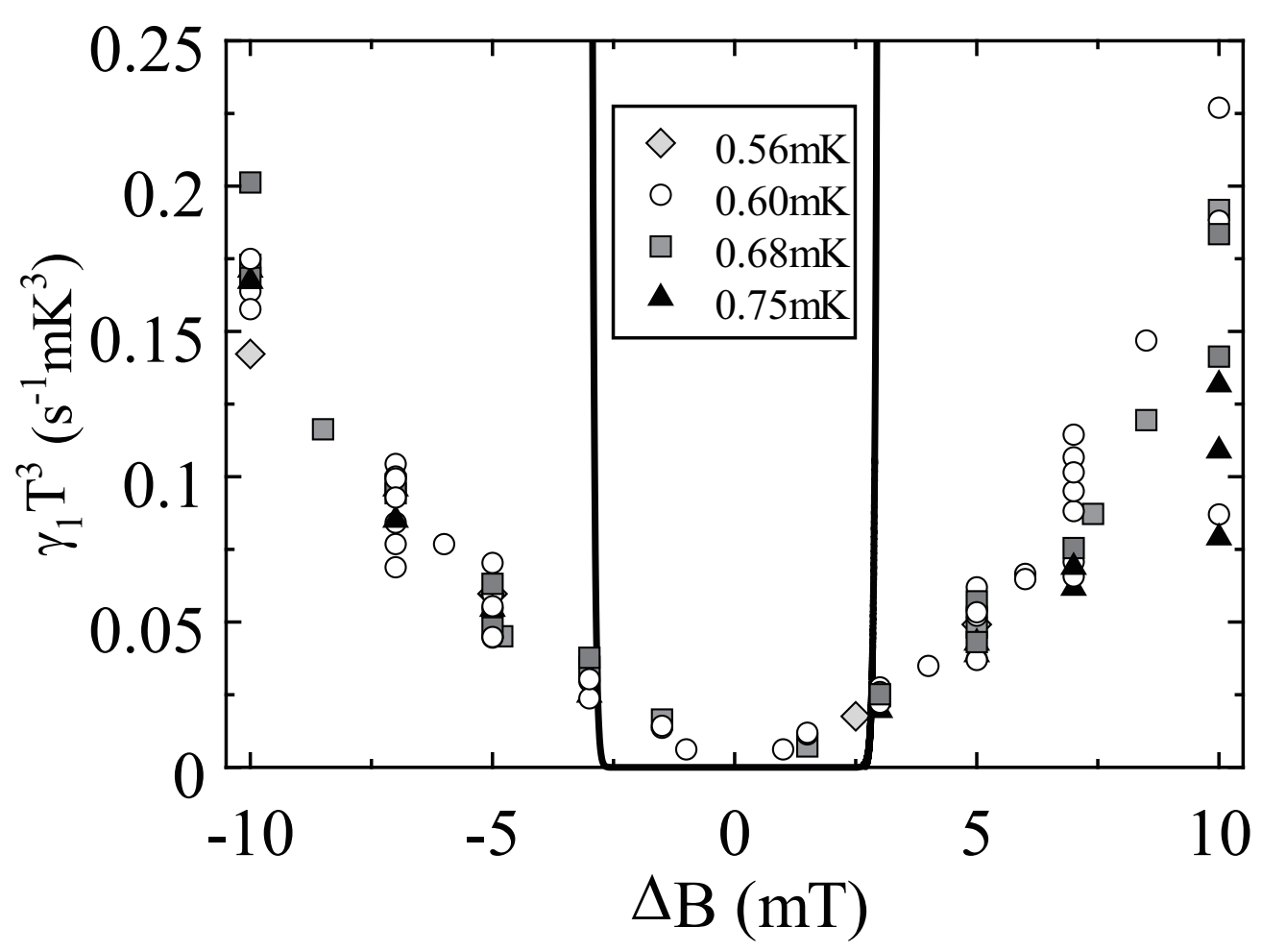

FIG. 6: The $\Delta B$ dependence of the $\gamma_{1} T^{3}$ at various temperatures. See text for the lines. 
(a) $\Delta$ B $>0$
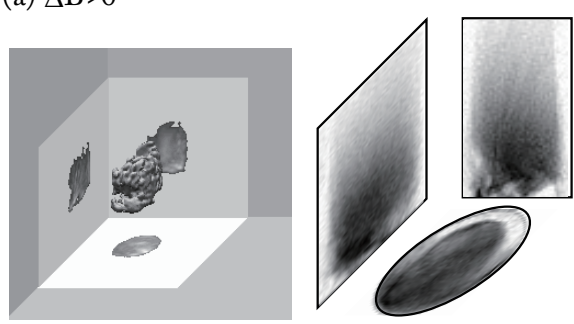

Before transition

(Left: 3D image, Right: 2D projections)

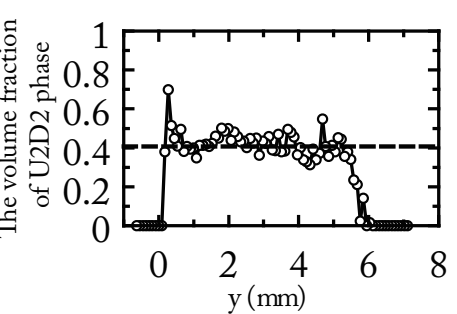

(b) $\Delta \mathrm{B}<0$
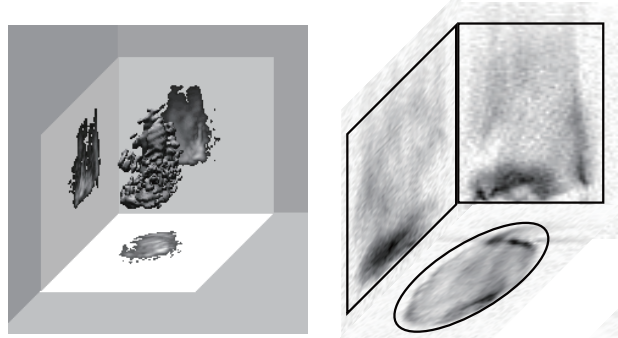

After transition

(Left: 3D image, Right: 2D projections)

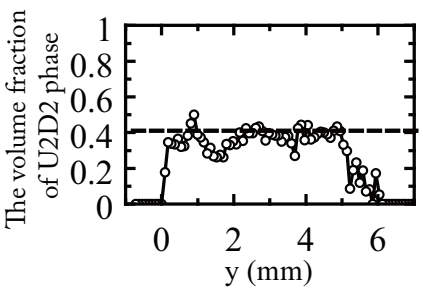

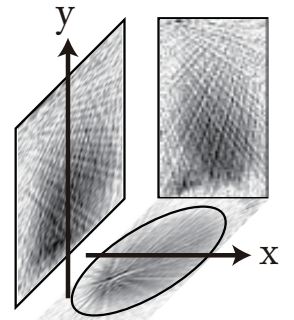

Soon after the first stage is terminated
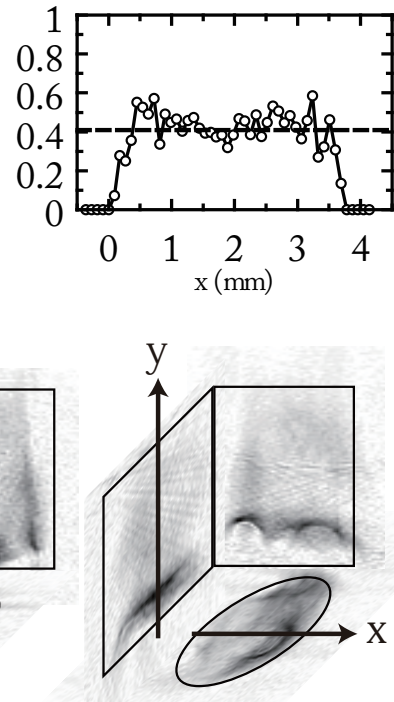

Soon after the first stage is terminated

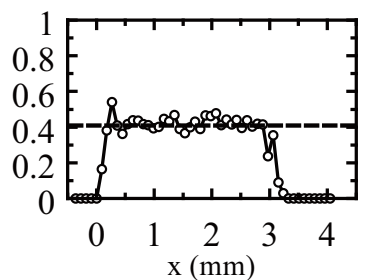

FIG. 7: The spatial distribution of U2D2 phase soon after the first stage is terminated. (a): $\Delta B>0$, the U2D2 phase is disappearing. (b): $\Delta B<0$, the U2D2 phase is appearing. The bottom graphs show the volume fraction of U2D2 phase to total crystal volume at the position along $\mathrm{x}$ or $\mathrm{y}$ axes. 


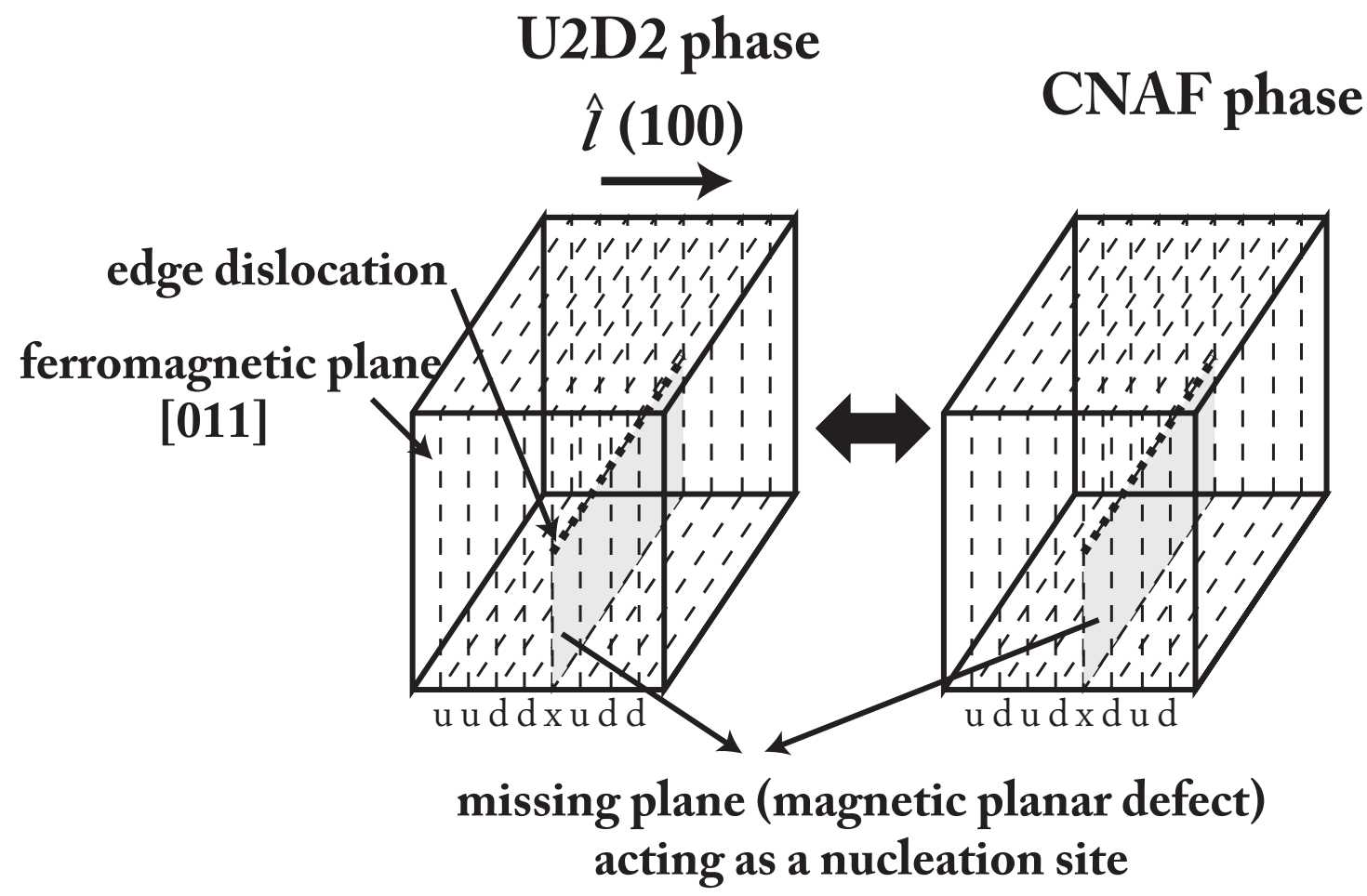

FIG. 8: Schematic illustration of a missing plane (grey hatching) labeled as x. 
(a) $\Delta \mathrm{B}<0$

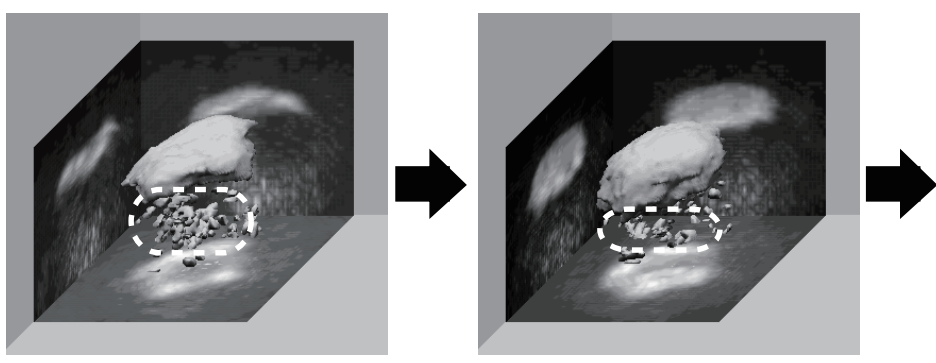

During the second stage

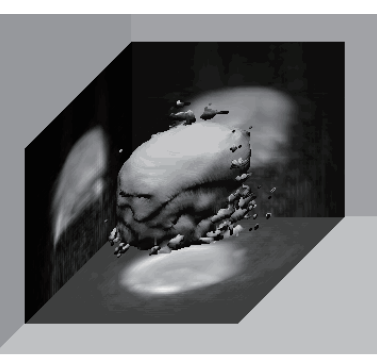

After transition

(b) $\Delta \mathrm{B}>0$

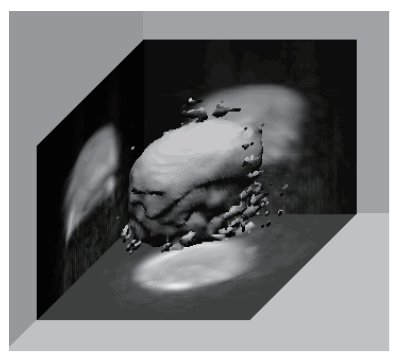

Before transition

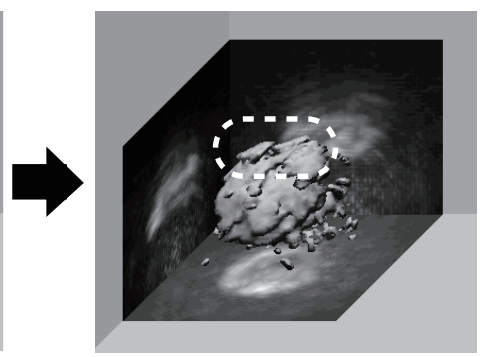

During the second stage

FIG. 9: The spatial distribution of U2D2 phase during the second stage. The upper right image shows an equilibrium shape of a solid in the U2D2 phase, which fills the lower half of the cylindrical cell, while the upper half is filled with liquid. Liquid-solid interface locates at the upper round surface of the image. (a): $\Delta B<0$, the U2D2 phase is appearing as faint flakes in the lower region indicated by a broken line. (b): $\Delta B>0$, the U2D2 phase is disappearing in the upper region indicated by a broken line. 